\title{
Cutaneous Filariasis in an American Traveler
}

\author{
Megan A. Jones-Sheets, DO; Michael Chen, MD; Julio C. Cruz, MD
}

\section{PRACTICE POINTS}

- Parasitic infections should always be a diagnostic consideration in individuals who present with a pruritic eruption and a history of travel.

- To control parasitic infections, research efforts should focus on improved sanitation, drug treatment, transmission prevention, and improved education of clinical manifestations to increase early detection.

To the Editor:

Cutaneous filariasis is a group of infectious diseases caused by more than 60 different nematode species and endemic to approximately 83 countries. ${ }^{1,2}$ These infections are transmitted to humans by vectors such as mosquitoes, blackflies, biting midges, or Tabanid flies. ${ }^{1}$ The blood meal taken by vectors allow microfilariae to enter the skin and develop into adult worms. ${ }^{1-3}$ It is postulated that filarial infections were first described in 2000 BC by Egyptian statues demonstrating elephantiasis, or swollen limbs, caused by chronic infections. ${ }^{1}$ Although there are numerous filarial nematode species that infect humans, each life cycle is similar. First, the arthropod vector transmits infective larvae through human skin during a blood meal. Then larvae migrate to host body parts where they mature into adults. Infective filariae require 1 to 2 weeks to form; arthropods may then have another blood meal and transmit the infection to another host. ${ }^{1}$

Filarial parasites have been categorized into groups based on the site of adult worm stage habitat. The cutaneous group includes Loa loa, Onchocerca volvulus, Mansonella perstans, and Dipetalonema streptocerca. The lymphatic group includes Wuchereria bancrofti, Brugia malayi, and Brugia timori. Lastly, the body cavity group includes Mansonella ozzardi. Because humidity is required for survival of the infective larval stage, individuals from tropical countries in Africa, Central America, and South America most commonly are affected. ${ }^{1}$ These diseases also are related to poor housing quality and inadequate sanitation. 1,3,4 Travel for business, medical missions, pleasure, or emigration has caused increased filarial infections globally. In fact, dermatologic disorders with infectious etiologies cause approximately $17 \%$ of travelers to seek medical attention. ${ }^{3}$

Dermatologic manifestations indicative of a potential cutaneous filarial infection include papules, nodules, excoriations with secondary xerosis, lichenification, skin pigment changes, and/or severe pruritus. However, individuals from filarial endemic regions may not demonstrate any clinical signs or symptoms, despite having microfilariae in their blood, ${ }^{1}$ which enables the disease to propagate and poses a major public health concern. In fact, patients with chronic filarial infections are at increased risk for developing lymphedema, elephantiasis, or blindness, which are hypothesized to be the second largest cause of permanent disability worldwide. ${ }^{1}$ Although rarely seen in US citizens, cutaneous filariasis should always be a diagnostic consideration in patients who present with a pruritic eruption and have a travel history to tropical countries. We report a case of cutaneous onchocerciasis in a US citizen who developed a pruritic eczematous eruption on the right upper arm following

Dr. Jones-Sheets is from Ohio University Heritage College of Osteopathic Medicine, Athens. Dr. Chen is from Skin Cancer and Dermatology of Columbus, Ohio. Dr. Cruz is from Buckeye Dermatology, Inc, Columbus.

The authors report no conflict of interest.

Correspondence: Megan A. Jones-Sheets, DO, 2044 Tremont Ave SW, Massillon, OH 44647 (mj822007@ohio.edu).

doi:10.12788/cutis.0101 
an arthropod assault while working in an Onchocerca endemic region approximately 1.5 years prior.

A 33-year-old woman presented to our outpatient dermatology clinic with the chief concern of a pruritic rash on the right arm (Figure 1). She revealed a history of travel to Peru, associated symptoms, prior diagnoses, and attempted treatment regimens. Over a 1.5-year period, the patient traveled for work-related reasons to Madre de Dios, Peru. During that time, the patient experienced 2 bouts of severe abdominal pain, nausea, and vomiting that she attributed to poor food and water quality. She did not immediately seek medical attention. She also developed skin manifestations that began as a pruritic and irritating pinpoint-sized red papule on the
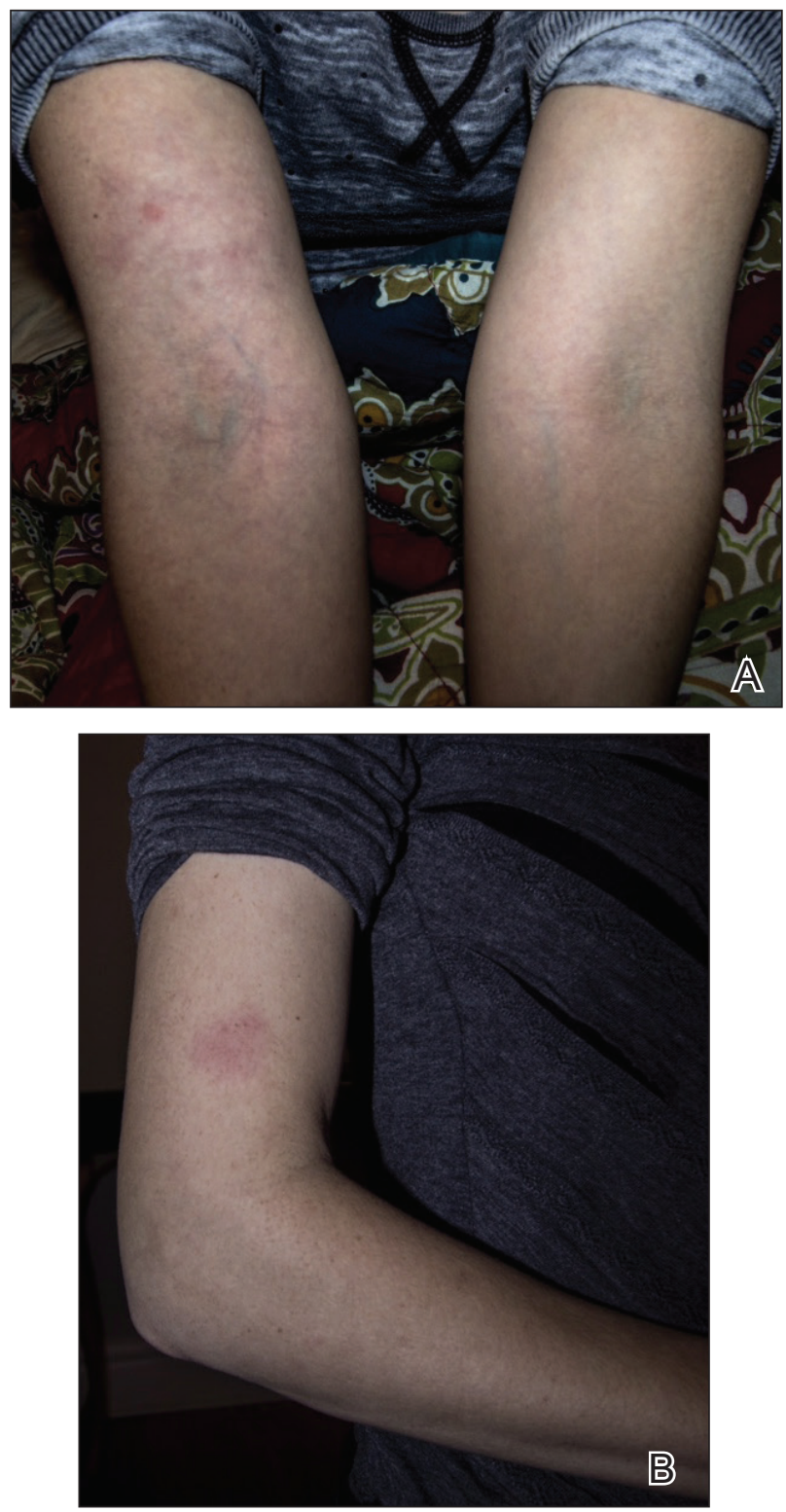

FIGURE 1. A, Erythematous patches on the right arm. B, A 1-cm subcutaneous nodule on the right elbow with overlying skin discoloration. right eyelid, possibly a site of an arthropod assault. She then experienced episodic eyelash loss (Figure 2) and subsequent vision changes, including blurry vision, halos around lights, and dimming around the periphery. When the patient developed circular, pink, pruritic plaques on the right upper extremity, she sought medical attention in Lima, Peru. She was diagnosed with tinea corporis and prescribed an oral antibiotic and topical antifungal.

This treatment regimen did not result in improvement. In the following months, the patient noticed worsening of the ocular symptoms, and she developed a dry cough with occasional dyspnea. She again sought medical attention in Peru and was referred to an ophthalmologist who suspected Demodex mite or fungal infection; however, workup for those pathologies was negative. The respiratory symptoms continued to worsen, particularly at night, and she began to experience palpitations.

Upon returning to the United States, the patient was evaluated by her primary care physician who ordered the following laboratory tests: a complete blood cell count with differential, comprehensive metabolic panel, vitamin D level, blood culture, lipid panel, ferritin level, and thyroid function. An electrocardiogram, throat culture, and stool culture for ova and parasites also were obtained. The electrocardiogram showed sinus tachycardia, and the stool culture revealed blastocystis, for which she was prescribed oral metronidazole and tinidazole. The other results were within reference range, and the throat culture showed normal oropharyngeal microbes.

A few days after the treatment for the blastocystis, the gastrointestinal tract symptoms improved, but dermatologic, ocular, pulmonary, and cardiac symptoms persisted. She also began experiencing night sweats and hot flashes. In between visits to the primary care physician, she sought medical attention at an urgent care clinic for worsening pulmonary and cardiac symptoms. At that time, results of a chest radiograph were normal.

The primary care physician referred her to infectious disease and dermatology for further evaluation within a few weeks of returning to the United States. Upon presentation to dermatology, the patient described a waxing and waning nature to the rash on the arm with associated intermittent pain. Physical examination revealed several erythematous patches on the triceps and a $1-\mathrm{cm}$

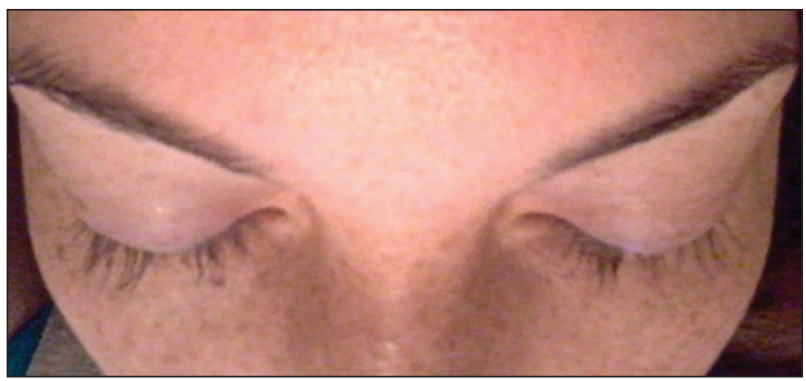

FIGURE 2. Clinically apparent eyelash loss. 
subcutaneous nodule on the right elbow with overlying skin discoloration. The patient stated that she first noticed the inflamed painful nodule when she returned to the United States. Since its discovery, the nodule increased in size and became firm. The patient also described occasional limited range of motion of the right arm due to the discomfort of the rash and inflamed nodule. Interestingly, the patient's partner who accompanied her to Peru also developed a similar pruritic rash on the chest. He was evaluated by dermatology and infectious disease; however, biopsies of his rash performed at a different practice revealed nonspecific results. Due to her partner's inconclusive results, our patient initially refused a biopsy; however, she returned to the office after 1 week with worsening symptoms, and a 4-mm punch biopsy of the right arm was obtained in addition to rapid plasma reagin and QuantiFERON-TB Gold test.

Based on the patient's travel history, clinical course, and physical examination, the clinical differential diagnosis included nummular dermatitis, panniculitis/nodular vasculitis, tinea corporis, secondary syphilis, pinta, tuberculosis, or cutaneous filariasis. Rapid plasma reagin and QuantiFERON-TB Gold test results were negative, and a periodic acid-Schiff stain of the biopsy was negative for fungal elements. Dermatopathology revealed intradermal filarial nematodes of 120 to $150 \mu \mathrm{m}$ in diameter and 1-to 2-mm cuticles eliciting a predominantly superficial and deep lymphohistiocytic reaction (Figure 3). The histopathologic differential diagnosis based on the diameter of the nematode included $M$ perstans, $W$ bancrofti, and $O$ volvulus. Clinical correlation was highly recommended to obtain a final diagnosis and management plan. A portion of the biopsy was sent to the Centers for Disease Control and Prevention, which confirmed the presence of a filarial nematode consistent with a zoonotic Brugia but also with zoonotic Onchocerca as a possible differential. Therefore, considering the history, clinical course, and histopathologic analysis, the final diagnosis of cutaneous onchocerciasis was made.

The patient was referred back to infectious disease and started on combination therapy of ivermectin $(25.5 \mathrm{mg})$ plus albendazole $(800 \mathrm{mg})$ taken at once followed by doxycycline $(200 \mathrm{mg})$ for 6 weeks. The symptoms initially worsened, then she experienced nearcomplete resolution of dermatologic, pulmonary, and ocular symptoms after approximately 2 weeks of treatment. She continued to report fatigue and palpitations, for she continued to see a cardiologist. We highly encouraged her to continue following up with infectious disease to ensure complete eradication of the infection.

Onchocerciasis is common in individuals who live or work in equatorial Africa, Central America, and South America.The disease process has dermatologic, ocular, and other systemic manifestations if the infection continues long-term without treatment. ${ }^{2,5}$ Although rare, this infection has been observed in US citizens who have traveled to filarial endemic regions for a period of time ranging from 2 weeks to 39 years. $2,6,7$
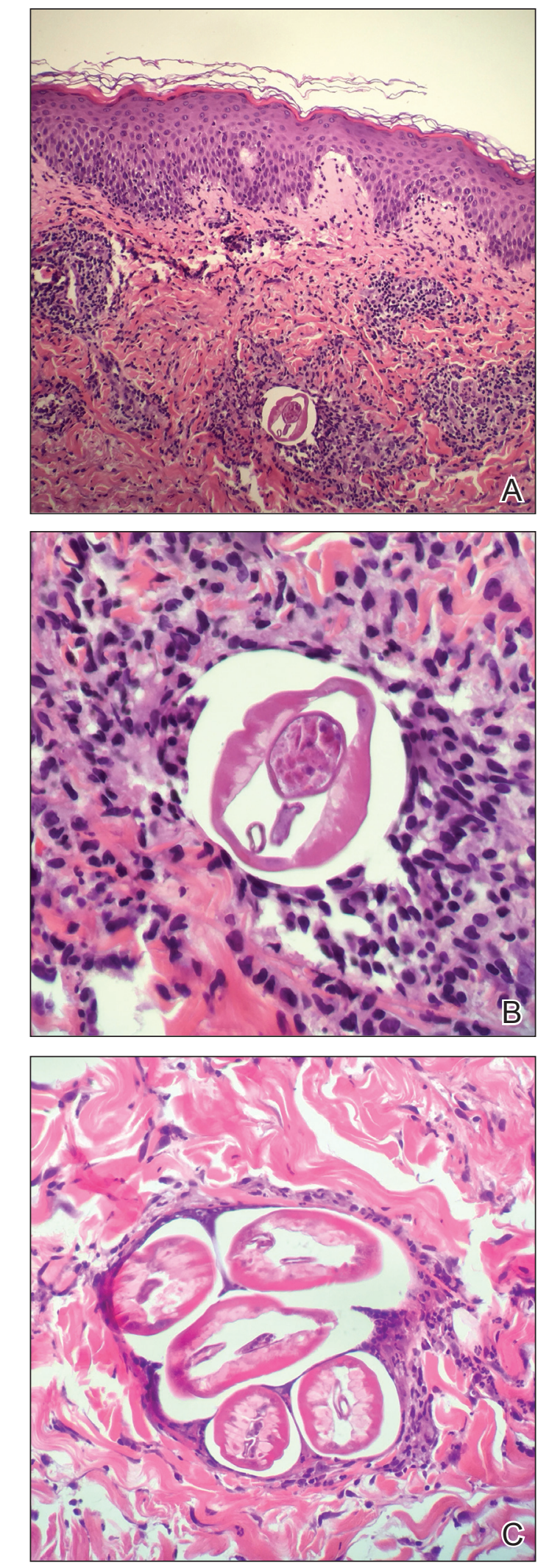

FIGURE 3. A-C, Histopathology demonstrated intradermal filarial nematodes with a superficial and deep lymphohistiocytic reaction (H\&E; original magnifications $\times 100, \times 400$, and $\times 400$, respectively). 
Blackflies (genus Simulium), the intermediate host of $O$ volvulus, breed in areas close to freely flowing water. The blackfly bites a human host, and within 7 days the microfilariae undergo 2 molts to reach the infective stage. The larvae remain in the dermis and subcutaneous tissue for 2 additional molts until they develop into adult worms. Then, adult worms may become encapsulated, eliciting and forming subcutaneous nodules, also known as onchocercomas. ${ }^{2,3}$

A fertilized female in the subcutaneous tissue releases microfilariae that may remain in the skin or travel to the eyes of the host. ${ }^{3}$ The host may demonstrate signs of infection within 3 to 15 months. ${ }^{2}$ Most commonly, patients report localized or generalized pruritus, and one extremity develops swelling, papules, lymphadenopathy, and alterations of skin pigment (hypopigmentation or hyperpigmentation)..$^{5-8}$ Our patient developed a pruritic eczematous eruption that only affected her right arm with an inflamed firm nodule overlying the elbow, a suspected onchocercoma. Onchocercomas are the direct result of adult worms coiled in the dermis, subcutaneous tissue, and deep fascia. These commonly are found in close proximity to bony prominences with the specific location pending the geographic area of infection. ${ }^{8}$ For example, onchocercomas more commonly are found over bony prominences in the head and upper body region in patients who acquired the disease from Central or South America, whereas individuals infected in Africa more commonly develop onchocercomas near the femoral, coccyx, or sacral regions. ${ }^{2}$

The immune response mounted by the infected host is responsible for the ocular signs and symptoms. ${ }^{2}$ Specifically, the inflammatory reaction may impact the patient's cornea, anterior uveal tract, chorioretinal zone, or optic nerve. In fact, onchocerciasis, or river blindness, is the leading cause of blindness in the world. ${ }^{2}$ The host immune response also is responsible for the dermatologic manifestations of this disease. In addition to the dermatologic manifestations already mentioned, others include epidermal atrophy, ulcerations, femoral or inguinal lymphadenitis, lymphedema, and/or general wasting in more severe long-standing infections. Additionally, patients may experience systemic signs resulting from an underlying $O$ volvulus infection. ${ }^{8}$ Our patient demonstrated a subjectively remarkable systemic response manifested by shortness of breath, dyspnea, cough, and palpitations, likely the result of her existing filarial infection.

Diagnosis of onchocerciasis is made by identification of microfilariae or adult worms in skin snips or punch biopsies. Histopathologic analysis of onchocerciasis has been described as several adult worms in the subcutaneous tissue surrounded by a granulomatous, fibrotic, calcified, or sometimes ossified inflammatory reaction. ${ }^{8}$ Microfilariae may migrate to the upper dermis and typically are surrounded by lymphocytes, histiocytes, plasma cells, and eosinophils with an absence of neutrophils, 5,8 which directly causes the overlying epidermis to undergo reactive changes. Measurement of filarial nematodes often helps differentiate species. For example, $O$ volvulus typically measures 230 to $500 \mathrm{~mm}$ in length for females and 16 to $42 \mathrm{~mm}$ in length for males. ${ }^{8}$ The thickness of cuticles typically measures 4 to $8 \mu \mathrm{m}$ for females and 3 to $5 \mu \mathrm{m}$ for males. Microfilariae of $O$ volvulus also have been identified in patient's sputum, urine, blood, and spinal fluid. ${ }^{8}$ An alternative diagnostic method described by Stingl et $\mathrm{al}^{9}$ is a diethylcarbamazine (DEC) patch test that was $92 \%$ accurate in onchocerciasis cases diagnosed by skin snip analysis. ${ }^{9}$ Therefore, this test may be a practical alternative when skin specimens are inconclusive.

Management of onchocerciasis should include excision of subcutaneous nodules to remove adult worms. In the past, DEC with suramin was prescribed and kills both microfilariae and adult worms. ${ }^{8}$ However, DEC may induce pruritus, chorioretinal damage, and optic neuritis and suramin is nephrotoxic. ${ }^{2}$ Therefore, oral ivermectin $(0.15-0.20 \mathrm{mg} / \mathrm{kg}$ one-time dose, may be repeated in 3-12 months) is supported to be a less harmful option. Patients treated with ivermectin have a decreased risk for transmitting infection to the blackfly vector for up to 6 months after treatment, and it is a more effective microfilaricidal agent than DEC., ${ }^{2,3}$ Oral doxycycline (100 mg/d for 6 weeks) commonly is added as adjuvant therapy because it kills Wolbachia species, a bacterium that is needed for $O$ volvulus reproduction. ${ }^{3}$

Although rare in the United States, cutaneous filariasis has become a prevalent public health concern, especially in tropical countries. Individuals with chronic cutaneous filarial infections are at increased risk for debilitating complications that negatively affect their quality of life and productivity. Although the World Health Organization has attempted to eradicate cutaneous filarial infections by mass drug administration, transmission of these diseases remains a challenge. Further research on treatment and methods to prevent transmission by controlling arthropod vectors is required to avoid short-term and long-term health consequences caused by cutaneous filariasis.

Parasitic infections should always be a diagnostic consideration in individuals who present with a pruritic eruption and a history of travel to foreign countries located in Africa, Central America, and South America. Dermatologists in the United States should increase familiarity with these infections because of increased travel, economic globalization, and the impact of global climate change on the geographic distribution of vector arthropods. To control these infections, research efforts should focus on improved sanitation; drug treatment; transmission prevention; and improved education of their clinical manifestations, especially mucocutaneous signs.

\section{REFERENCES}

1. Mendoza N, Li A, Gill A, et al. Filariasis: diagnosis and treatment. Dermatol Ther. 2009;22:475-490.

2. Maso MJ, Kapila R, Schwartz RA, et al. Cutaneous onchocerciasis. Int J Dermatol. 1987;26:593-596. 
3. Lupi O, Downing $\mathrm{C}$, Lee $\mathrm{M}$, et al. Mucocutaneous manifestations of helminth infections. J Am Acad Dermatol. 2015;73:929-944.

4. Bolivar-Meija A, Alarcon-Olave C, Rodriguez-Morales AJ. Skin manifestations of arthropod-borne infection in Latin America. Curr Opin. 2014;27:288-294.

5. Okulicz JF, Stibich AS, Elston DM, et al. Cutaneous onchocercoma. Int J Dermatol. 2004;43:170-172.

6. Nguyen JC, Murphy ME, Nutman TB, et al. Cutaneous onchocerciasis in an American traveler. Int Soc Dermatol. 2005;44:125-128.
7. Toovey S, Moerman F, van Gompel A. Special infectious disease risks of expatriates and long-term travelers in tropical countries part II: infections other than malaria. J Travel Med. 2007;14:50-60.

8. Meyers WM, Neafie RC, Connor DH. Bancroftian and malayan filariasis. In Binford $\mathrm{CH}$, ed. Pathology of Tropical and Extraordinary Diseases. Vol 2. Fort Sam Houston, TX: Armed Forces Institute of Pathology; 1976:340-355.

9. Stingl P, Ross M, Gibson DW, et al. A diagnostic "patch-test" for onchocerciasis using topical diethylcarbamazine. Trans R Soc Trop Med Hyg. 